\title{
Optimal control of a batch fermentation process with nonlinear time-delay and free terminal time and cost sensitivity constraint
}

\author{
Jinlong Yuan ${ }^{\mathrm{a}, \mathrm{b}, \mathrm{c}, *}$, Chongyang Liu ${ }^{\mathrm{d}}, \mathrm{Xu}$ Zhang $^{\mathrm{a}}$, Jun Xie ${ }^{\mathrm{e}}$, Enmin Feng $^{\mathrm{a}}$, Hongchao Yin ${ }^{\mathrm{b}}$, \\ Zhilong Xiu ${ }^{c}$ \\ ${ }^{a}$ School of Mathematical Sciences, Dalian University of Technology, Dalian 116024, Liaoning, PR China \\ ${ }^{b}$ School of Energy and Power Engineering, Dalian University of Technology, Dalian 116024, Liaoning, PR China \\ ${ }^{c}$ School of Life Science and Biotechnology, Dalian University of Technology, Dalian 116024, Liaoning, PR China \\ ${ }^{d}$ School of Mathematics and Information Science, Shandong Institute of Business and Technology, Yantai 264000, Shandong, China \\ ${ }^{e}$ Teaching and Research Office of Mathematics, Department of Basics, PLA Dalian Naval Academy, Dalian 116018, Liaoning, PR China
}

\begin{abstract}
In this paper, we consider a nonlinear time-delay dynamic system with uncertain system parameters to characterize the process of batch fermentation. Our goal is to design an optimal control scheme to maximize the productivity of 1,3- propanediol (1,3PD). Accordingly, we introduce an optimal control problem governed by the nonlinear time-delay dynamic system, in which the control variables are the free terminal time of the batch fermentation process and the initial concentrations of biomass and glycerol. The optimal control problem is subject to a cost sensitivity constraint for ensuring that an acceptable level of the system performance is achieved and continuous state inequality constraints for ensuring that the concentrations of biomass, glycerol, 1,3-PD, acetate, ethanol lie within specified limits. Then, the optimal control problem with free terminal time is transformed, via a hybrid time-scaling strategy, into an equivalent problem with fixed terminal time, which is much preferred for numerical computation. Using the constraint transcription and local smoothing approximation techniques, we approximate these continuous state inequality constraints by conventional inequality constraints to yield an approximate optimal control problem. Because of the highly complex nature of this approximate problem, a parallel algorithm based on the filled function method is constructed to solve this approximate problem. Finally, it is observed that the optimal control obtained is satisfactory through numerical simulations.
\end{abstract}

\section{(c) 2016 Published by Elsevier Ltd.}

Keywords: Nonlinear time-delay system, Optimal control, Free terminal time, Cost sensitivity constraint, Parallel optimization, Batch fermentation

\section{Introduction}

1,3-Propanediol (1,3-PD) is one of the important products with numerous applications, in particular for production of a new type of polyester, which is referred to as polytrimethylene terepthalate [1]. Currently, the process of producing 1,3-PD can be divided into two types: microbial conversion and chemical synthesis [2]. Compared with chemical synthesis approaches, microbial conversion methods have received attention recently because it is a choice for cheaply renewable feedstock, high region specificity and no harm to environment [3]. From both the economical

\footnotetext{
${ }^{*}$ Corresponding author

Email addresses: yuanjinlong0613@163.com (Jinlong Yuan), hcyin@dlut.edu.cn (Hongchao Yin)
} 
and ecological point of view, it is an effective method to produce 1,3-PD by glycerol bioconversion induced by Klebsiella pneumonia (K. pneumonia) [4]. At present, there are three microbial conversion methods for producing 1,3-PD: batch, continuous and fed-batch fermentations [5]. In batch fermentation, bacteria and substrate are presented at the beginning of the process, and nothing is added or removed from the fermentor during the process [6]. In continuous fermentation, fresh medium is added continuously to replenish consumed substrate while old medium is removed during the reaction [7, 8]. In fed-batch fermentation, glycerol and alkali are discontinuously added to the reactor at constant rates, so as to keep the substrate concentration and the $\mathrm{pH}$ in the desirable levels, without the removal of medium $[9,10]$. There are several reasons for the necessity of studying batch fermentation [11]: 1) in batch fermentation, 1,3-PD yield, which is defined as the ratio between the formation of 1,3-PD and the consumption of glycerol, is high. The main reason is that the batch fermentation can be expressed as an excessive metabolism process of glycerol. That is, the high concentration of glycerol leads to the high formation of 1,3-PD and the low formation of by-product in batch fermentation of glycerol. In particular, note that the high target product yield in batch fermentation is only applicable to the bioconversion of glycerol. Not all the batch fermentations are of the high target product yield; 2) batch fermentation is a simple and easy operation mode compared with fed-batch and continuous fermentations; 3 ) batch fermentation is basic for understanding or controlling fed-batch and continuous fermentations.

In the laboratory, it is impractical to carry out lots of batch experiments to obtain high production concentration and molar yield of 1,3-PD. Consequently, it is necessary to describe the process of batch fermentation through the application of mathematical systems. In [12], a substrate-sufficient kinetic system is proposed to characterize substrate (glycerol) consumption and extracellular substances (1,3-PD, acetate and ethanol) formation in batch fermentation. Robust identification of enzymatic nonlinear dynamical systems for 1,3-PD transport mechanisms in microbial batch fermentation is carried out in [13]. Modelling and pathway identification involving the transport mechanism of a complex metabolic system in batch fermentation is investigated in [14]. Robustness analysis and identification of nonlinear multi-stage dynamical system in batch fermentation are considered in $[15,16,17]$. Parameter identification for a nonlinear enzyme-catalytic dynamic system with multiple time-delays is studied in [18]. The different identification problems for nonlinear hybrid dynamical system in batch fermentation are investigated in [19, 20]. A stochastic model for microbial fermentation process under Gaussian white noise environment is considered in [21]. Joint estimation of batch fermentation is carried out by using unscented kalman filter in [22].

An optimal control problem, in which both process yield and process sensitivity are considered in the objective function, is investigated in [23]. However, there is one limitation: the presence of time-delay, which is always encountered in real-world applications, is ignored in [23]. In [24], a parameter identification for a nonlinear timedelay system is carried out in microbial batch fermentation. The optimal control problem governed by this nonlinear time-delay system is studied in [25]. However, a cost sensitivity corresponding to uncertainty system parameters has not yet been considered. Clearly, a nonlinear dynamic system is an idealized description about the actual behavior of a biological or engineering system. During the life span of the system, the values of some system parameters may change $[26,27]$. For an optimal control problem governed by a nonlinear dynamical system, the optimal cost function obtained is under the assumption that these system parameters of the dynamical system are fixed. Given some of these system parameters are subject to variation, the cost sensitivity corresponding to uncertainty system parameters should be taken into consideration [28]. Nonetheless, in the process of microbial fermentation, there are few papers dedicated to the cost sensitivity.

In this paper, we consider a nonlinear time-delay dynamic system to characterize the process of batch fermentation of glycerol bioconversion to 1,3-PD induced by $K$. pneumonia. Our aim is to construct an optimal control to maximize the productivity of 1,3-PD. Therefore, we propose an optimal control problem that depend on system parameters which have some uncertainty as to their exact values. The control variables are the terminal time of the batch fermentation process and the initial concentrations of biomass and glycerol. The optimal control problem governed by a nonlinear time-delay dynamic system, is subject to cost sensitivity (the derivative of the cost function with respect to the uncertain system parameters) constraint for ensuring that an acceptable level of the system performance is achieved and continuous state inequality constraints for ensuring that the concentrations of biomass, glycerol, and reaction products lie within specified limits. Then, the optimal control problem with free terminal time is transformed into an equivalent optimal control problem with fixed terminal time via a hybrid time-scaling strategy that only maps the current state into the new time scale and the delayed state remain in the original time scale. The equivalent problem is much preferred for numerical computation. Through the application of the constraint transcription and local smoothing approximation techniques, we approximate the continuous state inequality constraints by conventional in- 
equality constraints to yield an approximate optimal control problem. On account of the highly computing complexity of this approximate problem, the computational cost is very high. Thus, a parallel algorithm is proposed to solve it based on the filled function method. Finally, numerical results show that the obtained optimal control is satisfactory.

The remainder of this paper is organized as follows. In Section 2, a nonlinear time-delay dynamic system is formulated. In Section 3, an optimal control problem subject to a cost sensitivity constraint and continuous state inequality constraints is proposed. In Section 4, computational approaches are constructed to solve the optimal control problem. In Section 5, numerical results are presented. In Section 6, conclusions and trace the direction for future works are presented.

\section{Nonlinear time-delay dynamic system}

\section{Nomenclature}

- $I_{n}$ denotes the set $\{1,2, \ldots, n\}$.

- $\mathbb{R}_{+}$denotes the set of nonnegative real numbers.

- $\mathbb{R}$ denotes the set of real numbers.

- $A^{\mathrm{T}}$ denotes the transposition of matrix or vector $A$.

- $T \in\left[a_{3}, b_{3}\right]$ is the free terminal time for the fermentation process, where $a_{3}$ and $b_{3}$ are the minimal and the maximal time durations, respectively.

- $[0, T] \subset \mathbb{R}_{+}$, is the interval of reaction time.

- $x_{0}:=\left[x_{01}, \ldots, x_{05}\right]^{\mathrm{T}} \in \mathbb{R}^{5}$, is the initial state vector.

- $a_{1}$ and $b_{1}$ are the values of the minimal and the maximal the initial state $x_{01}$, respectively.

- $a_{2}$ and $b_{2}$ are the values of the minimal and the maximal the initial state $x_{02}$, respectively.

- $x(t):=\left[x_{1}(t), \ldots, x_{5}(t)\right]^{\mathrm{T}} \in \mathbb{R}^{5}$, denotes the continuous state vector (whose components are the state variables), where $x_{1}(t), \ldots, x_{5}(t)$ denote the concentrations of biomass, glycerol, 1,3-PD, acetate, ethanol at time $t \in[0, T]$, respectively.

- $\mu_{m}$ is the maximum specific growth rate.

- $k_{1}$ is the Monod saturation constant.

- $m_{2}$ is the maintenance term of substrate consumption under substrate-limited conditions.

- $Y_{2}$ is the maximum growth yields.

- $m_{i}, i=3,4,5$, are the maintenance terms of 1,3-PD, acetate and ethanol under substrate-limited conditions.

- $Y_{i}, i=3,4,5$, are the maximum 1,3-PD, acetate and ethanol yields.

In the actual experiment, the following conditions are assumed throughout, whether explicitly mentioned or not.

H 1. No medium is pumped inside and outside the bioreactor in the process of batch fermentation.

H 2. The concentrations of reactants are uniformly distributed in the reactor.

Under Assumptions $\mathbf{H 1}$ and $\mathbf{H 2}$, mass balance of biomass, substrate and products in batch fermentation can be described as the following nonlinear time-delay system [24],

$$
\left\{\begin{array}{l}
\dot{x}(t)=f(x(t), x(t-\tau), \sigma), t \in(0, T], \\
x(t)=\phi(t), \quad t \leq 0, \\
x(0)=x_{0},
\end{array}\right.
$$

where $\tau=0.26$ is a time-delay for biomass in the model of the fermentation process; $\phi(t)$ is a given initial function; $\sigma \in \mathbb{R}^{10}$ is uncertain system parameter vector (whose components are uncertain system parameters). Note that these uncertain system parameters are difficult to determine exactly. Methods for estimating the values of these uncertain system parameters using experimental data are given in [24]. We will use these estimates listed in Table 1 as the nominal values $\sigma^{0}$ of system parameter vector in this paper. 
Table 1. The nominal values $\sigma^{0}$ of system parameter vector for system (1) listed in [24].

\begin{tabular}{cccccc}
\hline$\sigma_{1}^{0}\left(h^{-1}\right)$ & $\sigma_{2}^{0}\left(\right.$ mmoll $\left.^{-1}\right)$ & $\sigma_{3}^{0}\left(\right.$ mmolg $\left.^{-1} h^{-1}\right)$ & $\sigma_{4}^{0}\left(\right.$ mmolg $\left.^{-1} h^{-1}\right)$ & $\sigma_{5}^{0}\left(\right.$ mmolg $\left.^{-1} h^{-1}\right)$ & $\sigma_{6}^{0}\left(\mathrm{mmolg}^{-1} h^{-1}\right)$ \\
\hline 0.994 & 0.368 & 3.24 & 3.679 & 0.491 & 7.309 \\
$\sigma_{7}^{0}\left(\right.$ mmolg $\left.^{-1}\right)$ & $\sigma_{8}^{0}\left(\right.$ mmolg $\left.^{-1}\right)$ & $\sigma_{9}^{0}\left(\mathrm{mmolg}^{-1}\right)$ & $\sigma_{10}^{0}\left(\right.$ mmolg $\left.^{-1}\right)$ & & \\
0.085 & 76 & 35.54 & 14.78 & \\
\hline \multicolumn{5}{c}{} \\
\hline
\end{tabular}

The dynamics of batch process is expressed by

$$
\left.f(x(t), x(t-\tau), \sigma):=\left[\mu(t) x_{1}(t-\tau), \sigma\right),-q_{2}(t) x_{1}(t-\tau), q_{3}(t) x_{1}(t-\tau), q_{4}(t) x_{1}(t-\tau), q_{5}(t) x_{1}(t-\tau)\right]^{\mathrm{T}} .
$$

In (2), the specific growth rate of cells can be defined by

$$
\mu(t)=\frac{\mu_{m} x_{2}(t)}{x_{2}(t)+k_{1}} \prod_{j=2}^{5}\left(1-\frac{x_{j}}{x_{j}^{*}}\right) .
$$

The specific consumption rate of substrate can be expressed by

$$
q_{2}(t)=m_{2}+\frac{\mu(t)}{Y_{2}} .
$$

The specific formation rates of 1,3-PD and acetic acid are defined by

$$
q_{i}(t)=-m_{i}+\mu(t) Y_{i}, i=3,4 \text {. }
$$

The specific formation rate of ethanol can be expressed by

$$
q_{5}(t)=m_{5}+\mu(t) Y_{5}
$$

Let $\sigma:=\left[\mu_{m}, k_{1}, m_{2}, m_{3}, m_{4}, m_{5}, Y_{2}, Y_{3}, Y_{4}, Y_{5}\right]^{\mathrm{T}} \in \mathbb{R}^{10}$.

Since each component of the state vector $x(t), t \in[0, T]$ represents a certain substance concentration, the concentrations of biomass, glycerol and products should be restricted in a certain range according to the practical fermentation process. Based on the results in [24], the admissible set $W$ of $x(t)$ is defined by

$$
x(t) \in W:=\left[x_{*}, x^{*}\right]=\prod_{i=1}^{5}\left[x_{* i}, x_{i}^{*}\right] \subset \mathbb{R}_{+}^{5},
$$

with $x_{*}=[0.01,0,0,0,0]^{\mathrm{T}}, x^{*}=[15,2039,939.5,1026,360.9]^{\mathrm{T}}$.

\section{Optimal control problem}

Let $x\left(\cdot \mid x_{01}, x_{02}, T, \sigma\right) \in \mathbb{R}^{5}$ denote the continuous solution of system (1) corresponding to the control variables $x_{01}, x_{02}, T$ and the system parameter vector $\sigma \in \mathbb{R}^{10}$. In batch fermentation, it is desired that the productivity of 1,3PD should be maximized at terminal time. This is achieved by manipulating the initial concentrations of biomass $x_{01}$ and glycerol $x_{02}$ and the free terminal time $T$. That is, the cost function is defined by

$$
J\left(x_{01}, x_{02}, T \mid \sigma\right)=\frac{x_{3}\left(T \mid x_{01}, x_{02}, T, \sigma\right)}{T} .
$$

In the process of microbial fermentation, we assumed that $\sigma$ is a given system parameter vector whose components are nominal estimates of the system parameters in Table 1. However, this assumption is idealistic since the nominal 
estimates are only approximations of the true system parameters. Therefore, inspired by the work in [26, 27, 28], we define the following cost sensitivity corresponding to the control variables $x_{01}, x_{02}, T$ as

$$
\left[\frac{\partial J\left(x_{01}, x_{02}, T \mid \sigma\right)}{\partial \sigma}\right]\left[\frac{\partial J\left(x_{01}, x_{02}, T \mid \sigma\right)}{\partial \sigma}\right]^{\mathrm{T}} .
$$

Clearly, formula (9) measures the rate at which the process yield changes with small variation of $\sigma$. Thus, a low value of the cost sensitivity indicates that the productivity of 1,3-PD is not sensitive to the variation of $\sigma$.

Let

$$
\begin{aligned}
g_{j}\left(x\left(t \mid x_{01}, x_{02}, T, \sigma\right)\right) & :=x_{j}\left(t \mid x_{01}, x_{02}, T, \sigma\right)-x_{j}^{*}, \forall t \in[0, T], j \in I_{5}, \\
g_{j+5}\left(x\left(t \mid x_{01}, x_{02}, T, \sigma\right)\right) & :=x_{j *}-x_{j}\left(t \mid x_{01}, x_{02}, T, \sigma\right), \forall t \in[0, T], j \in I_{5} .
\end{aligned}
$$

Therefore, in batch process, the optimal control problem can be formulated as

$$
\begin{aligned}
\text { Problem }(\mathbf{O C P}): \max & J\left(x_{01}, x_{02}, T \mid \sigma\right) \\
\text { s.t. } & {\left[\frac{\partial J\left(x_{01}, x_{02}, T \mid \sigma\right)}{\partial \sigma}\right]\left[\frac{\partial J\left(x_{01}, x_{02}, T \mid \sigma\right)}{\partial \sigma}\right]^{\mathrm{T}} \leq \epsilon, } \\
& g_{j}\left(x\left(t \mid x_{01}, x_{02}, T, \sigma\right)\right) \leq 0, \forall t \in[0, T], j \in I_{10}, \\
& \left(x_{01}, x_{02}, T\right) \in\left[a_{1}, b_{1}\right] \times\left[a_{2}, b_{2}\right] \times\left[a_{3}, b_{3}\right],
\end{aligned}
$$

where Inequation (11) is a cost sensitivity constraint for ensuring an acceptable level of the system performance is achieved, where $\epsilon$ is a given small constant; (12) is termed the continuous state inequality constraint [30], which must be satisfied at every point in the time horizon $[0, T]$.

For a given $\sigma \in \mathbb{R}^{10}$, any vector $\left(x_{01}, x_{02}, T\right) \in\left[a_{1}, b_{1}\right] \times\left[a_{2}, b_{2}\right] \times\left[a_{3}, b_{3}\right]$ satisfying constraints (11) and (12) is called an admissible control vector for system (1). Let $\mathcal{U}$ denote the set of all admissible control vectors. In view of the actual fermentation process, $\mathcal{U}$ is not an empty set. Therefore, we assume that

H 3. $\mathcal{U}$ is not an empty set.

From the definition of the set $\mathcal{U}$, it follows that we have the following property:

Property 1. Under Assumption $\mathbf{H 3}, \mathcal{U}$ is a compact set.

The existence of the optimal solution to Problem OCP is stated as follows.

Theorem 1. For a given $\sigma \in \mathbb{R}^{10}$, Problem OCP has an optimal solution.

Proof. From (8), we see that the cost function is continuous in $\left(x_{01}, x_{02}, T\right) \in \mathcal{U}$. Since the set $\mathcal{U}$ is compact, the proof follows readily.

\section{Computational approaches}

\subsection{Hybrid Time-scaling Transformation}

In Problem OCP, the direct method to calculating cost sensitivity defined in (9) involves one of the two gradient computation procedures (either the variational approach or the costate approach). It is cumbersome to integrate the state and variational or costate systems numerically when the terminal time $T$ is a decision variable to be optimized [29]. To address the problem caused by the free terminal time $T$, the time-scaling transformation is proposed in $[30,31]$. It is one of the most popular tools to transform Problem OCP into an equivalent problem with fixed terminal time. This time-scaling transformation affects the delay length in a complicated manner. In reality, under this timescaling transformation, a fixed time-delay in the original time horizon becomes a variable time-delay in the new time horizon. Consequently, in a way, this time-scaling transformation complicates, rather than simplifies, the computation of optimal control problems involving time-delay systems. In [37], a hybrid time-scaling strategy is proposed, where the current state vector is mapped into the new time horizon while the delayed state vector remains in the original time horizon. 
This transformation works by introducing a new time variable $s \in[-\tau, 1]$ and relating $s$ to $t$ through the equation

$$
t=\bar{\mu}(s \mid T)= \begin{cases}T, & \text { if } s=1, \\ T \cdot s, & \text { if } 0 \leq s<1, \\ s, & \text { if } s<0,\end{cases}
$$

where $\bar{\mu}$ is the so-called time-scaling function.

It is easy to verity that the time-scaling function is non-decreasing, continuous and piecewise-linear. Moreover,

$$
\frac{\mathrm{d} \bar{\mu}(s \mid T)}{\mathrm{d} s}=T, s \in(0,1) .
$$

Let $\tilde{x}(s):=x(\bar{\mu}(s \mid T))$. Then, applying the time-scaling transformation $t=\bar{\mu}(s \mid T)$ gives the following new differential equation:

$$
\left\{\begin{array}{l}
\dot{\tilde{x}}(s)=\frac{\mathrm{d}}{\mathrm{d} s}(\tilde{x}(s))=\frac{\mathrm{d}}{\mathrm{d} s}(x(\bar{\mu}(s \mid T)))=T \frac{\mathrm{d} x(\bar{\mu}(s \mid T))}{\mathrm{d} t}, s \in(0,1] \\
\tilde{x}(s)=\phi(s), s \leq 0 \\
\tilde{x}(0)=x_{0}, s=0 .
\end{array}\right.
$$

Note that the delayed argument $\bar{\mu}(s \mid T)-\tau$, depends on $s$ and $T$. Let $s_{\text {delay }}=s_{\text {delay }}(s, T)$ and $t_{\text {delay }}=t_{\text {delay }}(s, T)$ denote the delayed time points in the new time horizon $[0,1]$ and the original time horizon $[0, T]$, respectively. Then, the relationship between $s_{\text {delay }}$ and $t_{\text {delay }}$ is given by the following fundamental equation:

$$
\bar{\mu}\left(s_{\text {delay }} \mid T\right)=t_{\text {delay }}=\bar{\mu}(s \mid T)-\tau .
$$

Thus, for $s \geq 0$, we have

$$
x(t-\tau)=x(\bar{\mu}(s \mid T)-\tau)=x\left(\bar{\mu}\left(s_{\text {delay }} \mid T\right)\right)=\tilde{x}\left(s_{\text {delay }}\right) .
$$

Then, by substituting (1) into (18) and (16), we obtain

$$
\left\{\begin{array}{l}
\dot{\tilde{x}}(s)=\tilde{f}\left(\tilde{x}(s), \tilde{x}\left(s_{\text {delay }}\right), \sigma, T\right)=T f\left(\tilde{x}(s), \tilde{x}\left(s_{\text {delay }}\right), \sigma\right), \quad \bar{\mu}\left(s_{\text {delay }} \mid T\right) \in(0, T], s \in(0,1], \\
\tilde{x}(s)=\phi(s), s \leq 0, \\
\tilde{x}(0)=x_{0}, s=0 .
\end{array}\right.
$$

The difficulty of solving (19) is that $s_{\text {delay }}$ depends on $s$ and $T$.

When $\bar{\mu}\left(s_{\text {delay }} \mid T\right)<0$, it follows from (14) that $s_{\text {delay }}<0$, and thus

$$
s_{\text {delay }}=\bar{\mu}\left(s_{\text {delay }} \mid T\right)=\bar{\mu}(s \mid T)-\tau .
$$

When $\bar{\mu}\left(s_{\text {delay }} \mid T\right) \geq 0$, it follows from (14) and (17) that $s_{\text {delay }}$ satisfies the following equation:

$$
T \cdot s-\tau=T \cdot s_{\text {delay }} .
$$

To obtain value of the delayed state $\tilde{x}\left(s_{\text {delay }} \mid x_{01}, x_{02}, T, \sigma\right)$, the delay time $s_{\text {delay }}$ is needed to be found. Note that $s_{\text {delay }}$ is a variable, which is determined through solving (21) at each time point $s$. Thus, to calculate a single cost function value, it is required to solve this equation at least $T / h$ times, where $h$, which is usually taken as $1 / 3600$, is the maximum step length, when solving the time-delay differential equations. Clearly, it is computationally expensive to solve the new problem, defined on the new time horizon. Thus, another way is needed to get the value of $\tilde{x}\left(s_{\text {delay }} \mid x_{01}, x_{02}, T, \sigma\right)$ and then solve (21).

To overcome the difficulties of determining $s_{\text {delay }}$, explicitly, we use the following identity:

$$
\tilde{x}\left(s_{\text {delay }}\right)=x\left(\bar{\mu}\left(s_{\text {delay }} \mid T\right)\right)=x\left(t_{\text {delay }}\right) .
$$

Substituting (17) and (22) into (19) yields the following dynamic system:

$$
\left\{\begin{array}{l}
\dot{\tilde{x}}(s)=\tilde{f}\left(\tilde{x}(s), x\left(t_{\text {delay }}\right), \sigma, T\right)=T f\left(\tilde{x}(s), x\left(t_{\text {delay }}\right), \sigma, T\right), \quad t_{\text {delay }} \in(0, T], s \in(0,1], \\
\tilde{x}(s)=\phi(s), s \leq 0, \\
\tilde{x}(0)=x_{0}, s=0 .
\end{array}\right.
$$


Let $\tilde{x}\left(\cdot \mid x_{01}, x_{02}, T, \sigma\right)$ denote the solution of system (23) corresponding to the admissible triple $\left(x_{01}, x_{02}, T\right)$.

Since $t_{\text {delay }}$ (unlike $s_{\text {delay }}$ ) can be determined explicitly at all points by using the equation $t_{\text {delay }}:=\bar{\mu}(s \mid T)-\tau$, there is no difficulty in evaluating the delay in system (23). Nonetheless, the right-hand side of system (23) relies on two coupled time-delay systems - one defined on the original time scale, the other defined on the new time scale. Therefore, this new time-scaling transformation is a hybrid transformation that combines information of the new and the old time horizons.

For a given triple $\left(x_{01}, x_{02}, T\right) \in\left[a_{1}, b_{1}\right] \times\left[a_{2}, b_{2}\right] \times\left[a_{3}, b_{3}\right], \tilde{x}\left(\cdot \mid x_{01}, x_{02}, T, \sigma\right)$ can be determined as follows. By the initial condition of system (23), $\tilde{x}\left(s \mid x_{01}, x_{02}, T, \sigma\right)=\phi(s)$, for $s \leq 0$. For $s>0$, we need to determine $\tilde{x}\left(s \mid x_{01}, x_{02}, T, \sigma\right)$ solving system (23) numerically. When solving this differential equation, $x\left(t_{\text {delay }}\right)$ on the right-hand side of system (23) is obtained using the previous values of $\tilde{x}\left(s \mid x_{01}, x_{02}, T, \sigma\right)$. More specifically, to determine the state value at $t_{\text {delay }}:=\bar{\mu}(s \mid T)-\tau$, we first find the unique past time point $t_{\ell}$ on the original time horizon such that $t_{\ell} \leq t_{\text {delay }} \leq t_{\ell+1}$. Such knot points must exist because $\bar{\mu}(s \mid T)$ is continuous and strictly increasing on $(-\infty, 1]$. The value of $x\left(t_{\text {delay }}\right)$ can be obtained by interpolating the values of $x\left(t_{\ell}\right)$ and $x\left(t_{\ell+1}\right)$.

Correspondingly, formula (10) and Problem OCP are transformed as follows, respectively

$$
\begin{aligned}
\tilde{g}_{j}\left(\tilde{x}\left(s \mid x_{01}, x_{02}, T, \sigma\right)\right) & :=\quad \tilde{x}_{j}\left(s \mid x_{01}, x_{02}, T, \sigma\right)-x_{j}^{*}, \forall s \in[0,1], j \in I_{5}, \\
\tilde{g}_{j+5}\left(\tilde{x}\left(s \mid x_{01}, x_{02}, T, \sigma\right)\right) & :=\quad x_{j *}-\tilde{x}_{j}\left(s \mid x_{01}, x_{02}, T, \sigma\right), \forall s \in[0,1], j \in I_{5},
\end{aligned}
$$

and

$$
\begin{aligned}
\text { Problem } \widetilde{\mathbf{O C P}}: \max \quad & \tilde{J}\left(x_{01}, x_{02}, T \mid \sigma\right):=\frac{\tilde{x}_{3}\left(1 \mid x_{01}, x_{02}, T, \sigma\right)}{T} \\
\text { s.t. } \quad & {\left[\frac{\partial \tilde{J}\left(x_{01}, x_{02}, T \mid \sigma\right)}{\partial \sigma}\right]\left[\frac{\partial \tilde{J}\left(x_{01}, x_{02}, T \mid \sigma\right)}{\partial \sigma}\right]^{\mathrm{T}} \leq \epsilon, } \\
& \tilde{g}_{j}\left(\tilde{x}\left(s \mid x_{01}, x_{02}, T, \sigma\right)\right) \leq 0, \forall s \in[0,1], j \in I_{10}, \\
& \left(x_{01}, x_{02}, T\right) \in\left[a_{1}, b_{1}\right] \times\left[a_{2}, b_{2}\right] \times\left[a_{3}, b_{3}\right] .
\end{aligned}
$$

\subsection{Computing cost sensitivity}

We now derive a formula for the cost sensitivity of Problem $\widetilde{\mathbf{O C P}}$ in the following theorem.

\section{Theorem 2.}

$$
\left[\frac{\partial \tilde{J}\left(x_{01}, x_{02}, T \mid \sigma\right)}{\partial \sigma}\right]\left[\frac{\partial \tilde{J}\left(x_{01}, x_{02}, T \mid \sigma\right)}{\partial \sigma}\right]^{\mathrm{T}}=\sum_{k=1}^{10}\left\{\frac{\lambda_{3}^{k}\left(1 \mid x_{01}, x_{02}, T, \sigma\right)}{T}\right\}^{2}
$$

where $\lambda\left(s \mid x_{01}, x_{02}, T, \sigma\right)=\left(\lambda_{j}^{k}\left(s \mid x_{01}, x_{02}, T, \sigma\right)\right)_{5 \times 10} \in \mathbb{R}^{5 \times 10}, s \in[0,1]$, is the solution of the following auxiliary delaydifferential system:

$$
\begin{aligned}
\dot{\lambda}\left(s \mid x_{01}, x_{02}, T, \sigma\right)= & \frac{\partial \tilde{f}\left(\tilde{x}(s), x\left(t_{\text {delay }}\right), \sigma, T\right)}{\partial \tilde{x}} \lambda\left(s \mid x_{01}, x_{02}, T, \sigma\right)+\frac{\partial \tilde{f}\left(\tilde{x}(s), x\left(t_{\text {delay }}\right), \sigma, T\right)}{\partial x} \lambda\left(s_{\text {delay }} \mid x_{01}, x_{02}, T, \sigma\right) \\
& +\frac{\partial \tilde{f}\left(\tilde{x}(s), x\left(t_{\text {delay }}\right), \sigma, T\right)}{\partial \sigma}, \forall s \in[0,1]
\end{aligned}
$$

with

$$
\lambda\left(s \mid x_{01}, x_{02}, T, \sigma\right)=\mathbf{0} \in \mathbb{R}^{5 \times 10}, \quad \forall s \leq 0 .
$$

Proof. Let $\left(x_{01}, x_{02}, T\right) \in\left[a_{1}, b_{1}\right] \times\left[a_{2}, b_{2}\right] \times\left[a_{3}, b_{3}\right]$ be an admissible triple. By Theorem 3.2 in [36], we have

$$
\frac{\partial \tilde{x}_{j}\left(1 \mid x_{01}, x_{02}, T, \sigma\right)}{\partial \sigma_{k}}=\lambda_{j}^{k}\left(1 \mid x_{01}, x_{02}, T, \sigma\right), j \in I_{5}, k \in I_{10},
$$


where $\lambda\left(s \mid x_{01}, x_{02}, T, \sigma\right)=\left(\lambda_{j}^{k}\left(s \mid x_{01}, x_{02}, T, \sigma\right)\right)_{5 \times 10} \in \mathbb{R}^{5 \times 10}$ is the solution of the systems (30) and (31). Thus, differentiating $\tilde{J}\left(x_{01}, x_{02}, T \mid \sigma\right)$ with respect to $\sigma_{k}$ yields

$$
\frac{\partial \tilde{J}\left(x_{01}, x_{02}, T \mid \sigma\right)}{\partial \sigma_{k}}=\frac{\partial}{\partial \sigma_{k}}\left\{\frac{\tilde{x}_{3}\left(1 \mid x_{01}, x_{02}, T, \sigma\right)}{T}\right\}=\frac{1}{T} \frac{\partial \tilde{x}_{3}\left(1 \mid x_{01}, x_{02}, T, \sigma\right)}{\partial \sigma_{k}}=\frac{\lambda_{3}^{k}\left(1 \mid x_{01}, x_{02}, T, \sigma\right)}{T}, k \in I_{10} .
$$

Hence,

$$
\left[\frac{\partial \widetilde{J}\left(x_{01}, x_{02}, T \mid \sigma\right)}{\partial \sigma}\right]\left[\frac{\partial \widetilde{J}\left(x_{01}, x_{02}, T \mid \sigma\right)}{\partial \sigma}\right]^{\mathrm{T}}=\sum_{k=1}^{10}\left\{\frac{\partial \widetilde{J}\left(x_{01}, x_{02}, T \mid \sigma\right)}{\partial \sigma_{k}}\right\}^{2}=\sum_{k=1}^{10}\left\{\frac{\lambda_{3}^{k}\left(1 \mid x_{01}, x_{02}, T, \sigma\right)}{T}\right\}^{2} .
$$

\subsection{Approximate problem}

Problem $\widetilde{\mathbf{O C P}}$ involves an infinite number of constraints (i.e. the continuous state inequality constraints imposed at every point in $[0,1]($ see $(27)))$. Thus, Problem $\widetilde{\mathbf{O C P}}$ can be viewed as a semi-infinite mathematical programming problem. The constraint transcription in conjunction with local smoothing approximation techniques are used to solve this type of optimal control problem in [30]. We will briefly discuss the application of these techniques to Problem $\widetilde{\text { OCP. }}$.

Constraints (27) can be transcribed equivalently to

$$
G^{j}\left(x_{01}, x_{02}, T \mid \sigma\right)=0, j \in I_{10},
$$

where $G^{j}\left(x_{01}, x_{02}, T \mid \sigma\right):=\int_{0}^{1} \max \left\{0, \tilde{g}_{j}\left(\tilde{x}\left(s \mid x_{01}, x_{02}, T, \sigma\right)\right)\right\} \mathrm{d} s, j \in I_{10}$. However, $G^{j}\left(x_{01}, x_{02}, T \mid \sigma\right), j \in I_{10}$, are nonsmooth in $\left(x_{01}, x_{02}, T\right) \in\left[a_{1}, b_{1}\right] \times\left[a_{2}, b_{2}\right] \times\left[a_{3}, b_{3}\right]$. Hence, we replace (32) with the following functional inequality constraints.

$$
\tilde{G}^{j}\left(x_{01}, x_{02}, T \mid \sigma\right):=\int_{0}^{1} \varphi_{\varepsilon, j}\left(\tilde{g}_{j}\left(\tilde{x}\left(s \mid x_{01}, x_{02}, T, \sigma\right)\right)\right) \mathrm{d} s \geq \gamma, j \in I_{10},
$$

where $\varepsilon>0, \gamma>0$ are adjustable parameters and

$$
\varphi_{\varepsilon, j}\left(\tilde{g}_{j}\left(\tilde{x}\left(s \mid x_{01}, x_{02}, T, \sigma\right)\right)\right):= \begin{cases}\tilde{g}_{j}\left(\tilde{x}\left(s \mid x_{01}, x_{02}, T, \sigma\right)\right), & \text { if } \tilde{g}_{j}\left(\tilde{x}\left(s \mid x_{01}, x_{02}, T, \sigma\right)\right) \in(\varepsilon,+\infty), \\ \frac{\left[\tilde{g}_{j}\left(\tilde{x}\left(s \mid x_{01}, x_{02}, T, \sigma\right)\right)+\varepsilon\right]^{2}}{4 \varepsilon}, & \text { if } \tilde{g}_{j}\left(\tilde{x}\left(s \mid x_{01}, x_{02}, T, \sigma\right)\right) \in[-\varepsilon, \varepsilon], \\ 0, & \text { if } \tilde{g}_{j}\left(\tilde{x}\left(s \mid x_{01}, x_{02}, T, \sigma\right)\right) \in(-\infty,-\varepsilon) .\end{cases}
$$

Hence, with this approximation scheme, constraints (27) in Problem $\widetilde{\mathbf{O C P}}$ are approximated by constraints (33), which are standard constraints and can be readily handled using the computational method described in Section 4.4. Then, by virtue of the results in [30], we obtain an approximate problem of Problem $\widetilde{\mathbf{O C P}}$ as given below.

$$
\begin{aligned}
& \text { Problem } \widetilde{\text { NOCP }}: \quad \max \quad \tilde{J}\left(x_{01}, x_{02}, T \mid \sigma\right)=\frac{\tilde{x}_{3}\left(1 \mid x_{01}, x_{02}, T, \sigma\right)}{T} \\
& \text { s.t. }\left[\frac{\partial \tilde{J}\left(x_{01}, x_{02}, T \mid \sigma\right)}{\partial \sigma}\right]\left[\frac{\partial \tilde{J}\left(x_{01}, x_{02}, T \mid \sigma\right)}{\partial \sigma}\right]^{\mathrm{T}} \leq \epsilon, \\
& \tilde{G}^{j}\left(x_{01}, x_{02}, T \mid \sigma\right) \geq \gamma, j \in I_{10}, \\
& \left(x_{01}, x_{02}, T\right) \in\left[a_{1}, b_{1}\right] \times\left[a_{2}, b_{2}\right] \times\left[a_{3}, b_{3}\right] .
\end{aligned}
$$

\subsection{A parallel filled function algorithm}

Since system (23) is highly nonlinear, Problem $\widetilde{\text { NOCP }}$ is too complicated to solve analytically. Problem $\widetilde{\text { NOCP }}$ is a smooth problem that can be solved using the gradient-based algorithms (see, for example, [32]). However, these techniques are designed to search for local optimal solutions. 
The main difficulty for global optimization is to escape from the current local minimizer and to find a better one. One of the most popular approaches is the filled function method, the conception of which was first proposed by Ge at the Dundee Biennial Conference on Numerical Analysis in 1983 and finally published in [33]. However, the traditional filled function methods are often non-differentiable and sensitive to some adjustable parameters, or contain ill-conditioned terms and more than one adjustable parameter needing to be controlled [35]. To overcome these limitations, a new filled function method is proposed in [34].

Because of the highly complex nature of Problem $\widehat{\text { NOCP }}$, we design a parallel filled function approach. On each slave processor, we can construct a filled function. Before the next iteration, on the master processor, we can obtain the optimal triple $\left(x_{01}, x_{02}, T\right)$ and use it to update the current triple $\left(x_{01}, x_{02}, T\right)$ on each slave processor. Then, on each slave processor, we can sequentially compute until the algorithm has a successful exit.

For convenience, Problem NOCP can now be equivalently transformed by the following optimal control problem called Problem $\overline{\mathbf{N O C P}}$

$$
\begin{aligned}
\text { Problem } \overline{\text { NOCP }: ~} \min \quad & \tilde{\mathcal{J}}\left(x_{01}, x_{02}, T \mid \sigma\right):=-\frac{\tilde{x}_{3}\left(1 \mid x_{01}, x_{02}, T, \sigma\right)}{T} \\
\text { s.t. } \quad & {\left[\frac{\partial \tilde{\mathcal{J}}\left(x_{01}, x_{02}, T \mid \sigma\right)}{\partial \sigma}\right]\left[\frac{\partial \tilde{\mathcal{J}}\left(x_{01}, x_{02}, T \mid \sigma\right)}{\partial \sigma}\right]^{\mathrm{T}} \leq \epsilon, } \\
& \tilde{G}^{j}\left(x_{01}, x_{02}, T \mid \sigma\right) \geq \gamma, j \in I_{10}, \\
& \left(x_{01}, x_{02}, T\right) \in\left[a_{1}, b_{1}\right] \times\left[a_{2}, b_{2}\right] \times\left[a_{3}, b_{3}\right] .
\end{aligned}
$$

The parameters in Algorithm 1 are defined below.

- Let $u$ denote $\left(x_{01}, x_{02}, T\right)$.

- $p$ is the slave processor index.

- $\tilde{N}$ is the number of slave processors.

- $k$ is the iteration index.

- $\mathcal{K}$ is the maximal iteration of $k$.

- $\delta$ is a nonnegative adjustable parameter satisfying

$0<\delta<\min \left\{\frac{1}{L^{2}}, \min \left\{\tilde{\mathcal{T}}\left(u_{p}^{k} \mid \sigma\right)-\tilde{\mathcal{J}}\left(\hat{u}_{p}^{k} \mid \sigma\right): \hat{u}_{p}^{k} \in S_{2}\right.\right.$ is a minimizer of $\tilde{\mathcal{J}}(u \mid \sigma)$ in $\left.\left.(35)\right\}\right\}, 0<L<\infty$ is a constant, with

$$
\begin{gathered}
S_{2} \leftarrow S_{21} \bigcup S_{22}, \\
S_{21} \leftarrow\left\{u \in\left[a_{1}, b_{1}\right] \times\left[a_{2}, b_{2}\right] \times\left[a_{3}, b_{3}\right] \mid \tilde{J}\left(u_{p}^{k} \mid \sigma\right)>\tilde{J}(u \mid \sigma)>\tilde{J}\left(u_{p}^{k} \mid \sigma\right)-\delta\right\}
\end{gathered}
$$

and

$$
S_{22} \leftarrow\left\{u \in\left[a_{1}, b_{1}\right] \times\left[a_{2}, b_{2}\right] \times\left[a_{3}, b_{3}\right] \mid \tilde{J}(u \mid \sigma)<\tilde{J}\left(u_{p}^{k} \mid \sigma\right)-\delta\right\} .
$$

- $A$ is a nonnegative adjustable parameter controlling the accuracy of the filled function method.

- $A_{\max }$ is an upper bound of parameter $A$.

- $\delta_{\min }$ is a lower bound of parameter $\delta$.

- $\widetilde{\mathcal{K}}$ is an integer for testing convergence (if the value of the cost function has not changed after $\widetilde{\mathcal{K}}$ iterations, then we terminate the algorithm).

- $\epsilon$ is the convergence tolerance of a cost sensitivity constraint for ensuring an acceptable level of the system performance is achieved.

- $\tilde{\epsilon}$ is the convergence tolerance of the cost function. 
- $u_{g}^{k}$ is the best control variables found by any member of the slave processor set at the end of the $k$ th iteration.

- $\tilde{J}\left(u_{g}^{k}\right)$ is the value of the cost function found by any member of the slave processor set at the end of the $k$ th iteration.

- $u_{p}^{k}$ is the control variables found by the $p$ th member of the slave processor set at the end of the $k$ th iteration.

- $\tilde{J}\left(u_{p}^{k}\right)$ is the value of the cost function found by the $p$ th member of the slave processor set at the end of the $k$ th iteration.

\section{Algorithm 1:}

Step 1 : Initialize and broadcast data.

- Step 1.1 : Read known data such as $\epsilon, \tilde{\epsilon}, \widetilde{\mathcal{K}}, \tilde{N}, W, a_{1}, b_{1}, a_{2}, b_{2}, a_{3}, b_{3}$ and $\mathcal{K}$ on master processor.

- Step 1.2 : Broadcast (MPI_Broad) data on master processor to all slave processors.

Step 2: Execute the following procedure on each slave processor and denote the ID of process as $p \in I_{\tilde{N}}$.

- Step 2.1 : Choose $u_{p}^{1} \in\left[a_{1}, b_{1}\right] \times\left[a_{2}, b_{2}\right] \times\left[a_{3}, b_{3}\right], \delta_{\min } \leftarrow 10^{-10}, A_{\max } \leftarrow 10^{5}, k \leftarrow 1$.

- Step 2.2 : Set $A \leftarrow 10, \delta \leftarrow 10^{-5}, \hbar_{1} \leftarrow 0, \hbar_{2} \leftarrow 0$. If $k>\mathcal{K}$, then the algorithm has an abnormal exit.

- Step 2.3 : Check and adjust.

1. Based on Theorem 2 and formula (33), check the values of $\left[\frac{\partial \tilde{\mathcal{T}}\left(u_{p}^{k} \mid \sigma\right)}{\partial \sigma}\right]\left[\frac{\partial \tilde{\mathcal{T}}\left(u_{p}^{k} \mid \sigma\right)}{\partial \sigma}\right]^{\mathrm{T}}$ and $\tilde{G}^{j}\left(u_{p}^{k} \mid \sigma\right), j \in I_{10}$.

2. If $\left[\frac{\partial \mathcal{\mathcal { T }}\left(u_{p}^{k} \mid \sigma\right)}{\partial \sigma}\right]\left[\frac{\partial \mathcal{\mathcal { T }}\left(u_{p}^{k} \mid \sigma\right)}{\partial \sigma}\right]^{\mathrm{T}}>\epsilon$ or $\exists j \in I_{10}$ such that $\tilde{G}^{j}\left(u_{p}^{k} \mid \sigma\right)<\gamma$ (i.e., $u_{p}^{k}$ is infeasible for Problem $\overline{\mathbf{N O C P}})$, then perform a gradient-descend search (minimizing $\left[\frac{\partial \tilde{\mathcal{J}}\left(u_{p}^{k} \mid \sigma\right)}{\partial \sigma}\right]\left[\frac{\partial \tilde{\mathcal{T}}\left(u_{p}^{k} \mid \sigma\right)}{\partial \sigma}\right]^{\mathrm{T}}$ ) or a gradientascent search (maximizing $\left.\tilde{G}^{j}\left(u_{p}^{k} \mid \sigma\right)\right)$ to obtain a feasible point $\breve{u}_{p}^{k}$ for Problem $\overline{\mathbf{N O C P}}\left(\right.$ i.e., $\left[\frac{\partial \tilde{\mathcal{J}}\left(\breve{u}_{p}^{k} \mid \sigma\right)}{\partial \sigma}\right]\left[\frac{\partial \tilde{\mathcal{T}}\left(\breve{u}_{p}^{k} \mid \sigma\right)}{\partial \sigma}\right]^{\mathrm{T}} \leq$ $\epsilon$ and $\left.\forall j \in I_{10}, \tilde{G}^{j}\left(\breve{u}_{p}^{k} \mid \sigma\right) \geq \gamma\right)$ and set $u_{p}^{k} \leftarrow \breve{u}_{p}^{k}$.

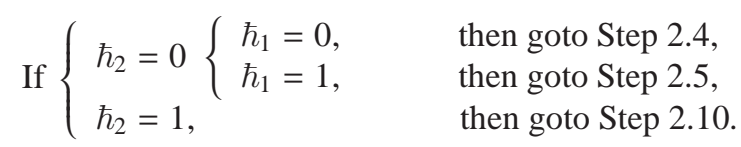

- Step 2.4 : Use the gradient-based algorithms with initial value $u_{p}^{k}$ to obtain local optimal solution of Problem $\overline{\mathbf{N O C P}}$, still denoted by itself $u_{p}^{k}$, set $\hbar_{1} \leftarrow 1$ and goto Step 2.3 .

- Step 2.5 : If $A>A_{\max }$, then $u_{p}^{k}$ is taken as a global minimizer of $\tilde{\mathcal{J}}(u \mid \sigma)$ in (35), set $\hbar_{2} \leftarrow 1$ and goto Step 2.3; otherwise, goto Step 2.6.

- Step 2.6 : Based on [34], for $u \in\left[a_{1}, b_{1}\right] \times\left[a_{2}, b_{2}\right] \times\left[a_{3}, b_{3}\right]$, construct

$$
F F_{u_{p}^{k}, A, \delta}(u) \leftarrow \min \left\{-\left\|u-u_{p}^{k}\right\|^{2}-\frac{\tilde{\mathcal{T}}^{2}(u \mid \sigma)}{A}, \min \left\{A\left(\tilde{\mathcal{T}}(u \mid \sigma)-\tilde{\mathcal{T}}\left(u_{p}^{k} \mid \sigma\right)\right),-\delta\right\}\left(\tilde{\mathcal{T}}(u \mid \sigma)-\tilde{\mathcal{T}}\left(u_{p}^{k} \mid \sigma\right)\right)^{2}\right\} .
$$

- Step 2.7 : Minimize $F F_{u_{p}^{k}, A, \delta}(u)$ from $2|u|$ initial points, where $|u|$ is the dimension of vector $u$. Let $v^{*}$ denote the minimizer of $F F_{u_{p}^{k}, A, \delta}(u)$.

- Step 2.8 : If $\tilde{\mathcal{J}}\left(v^{*} \mid \sigma\right)<\tilde{\mathcal{J}}\left(u_{p}^{k} \mid \sigma\right)$, then set $u_{p}^{k} \leftarrow v^{*}$, goto Step 2.6; otherwise, goto Step 2.9.

- Step 2.9 : Let $\delta \leftarrow 0.1 \delta$. If $\delta>\delta_{\min }$, goto Step 2.5; otherwise, let $A \leftarrow 10 A$ and goto Step 2.5. 
- Step 2.10 : Compute the cost function (35), denoted by $\tilde{\mathcal{J}}\left(u_{p}^{k} \mid \sigma\right)$. Send (MPI_Send) $\tilde{\mathcal{T}}\left(u_{p}^{k} \mid \sigma\right)$ and $u_{p}^{k}$ to master processor and Barrier (MPI_Barrier) until all slave processors complete the work in the $k$ th iteration.

Step 3 : Assign the master processor to execute the tasks: receive (MPI_Recv) $\tilde{\mathcal{J}}\left(u_{p}^{k} \mid \sigma\right)$ and $u_{p}^{k}$ from the $p$ th processor; compute

$$
\tilde{\mathcal{J}}\left(u_{g}^{k}\right) \leftarrow \min _{p=1, \ldots, \tilde{N}} \tilde{\mathcal{J}}\left(u_{p}^{k} \mid \sigma\right), \quad u_{g}^{k} \leftarrow \arg \min _{p=1, \ldots, \tilde{N}} \tilde{\mathcal{J}}\left(u_{p}^{k} \mid \sigma\right) .
$$

Then, Broadcast (MPI_Broad) $u_{g}^{k}$ to all slave processors and set $\tilde{\mathcal{J}}_{\text {best }}^{k} \leftarrow \tilde{\mathcal{J}}\left(u_{g}^{k}\right)$.

Step 4 : Execute the following procedure on each slave processor: based on broadcasted $u_{g}^{k}$ in master processor, obtain $u_{g}^{k}$; then, use $u_{g}^{k}$ to replace $u_{p}^{k+1}$.

Step 5 : Assign the master processor to execute the tasks: If $k \geq \widetilde{\mathcal{K}},\left|\tilde{\mathcal{T}}_{\text {best }}^{k-\widetilde{\mathcal{K}}+1}-\tilde{\mathcal{J}}_{\text {best }}^{k}\right|<\tilde{\epsilon}$, then report results and the algorithm has a successful exit; otherwise, set $k \leftarrow k+1$ and goto Step 2.2.

Remark 1. In Step 2.2 and Step 5, the algorithm stops when any of the following conditions holds:

1. The maximal iteration $\mathcal{K}$ is reached;

2. The deviation between the best fitness of current iteration and that of the $\widetilde{\mathcal{K}}$ th previous iteration is less than $\tilde{\epsilon}$.

Remark 2. Recall from Theorems 2.1 and 2.3 in [34] that the value of A should be selected large enough and the value of $\delta$ should be selected small enough. Otherwise, there could be no minimizer of $F F_{u_{p}^{k}, A, \delta}(u)$ in a better basin.

1. The value of $\delta$ should be selected small enough. We set the initial adjustable parameter $\delta$ of $10^{-5}$ in Step 2.2. Thus, the value of $\delta$ is reduced successively in Step 2.9 when certain step fails. If the adjustable parameter $\delta$ reaches its lower bound and we cannot find the better local minimizer, we increase the value of $A$.

2. The value of A should be selected large enough. We set the initial adjustable parameter A of 10 in Step 2.2. Afterwards, the value of $A$ is increased successively in Step 2.9 if no better solution is found when minimizing the filled function. If the adjustable parameter A reaches its upper bound and we cannot find the better local minimizer, the algorithm is terminated and the current local minimizer is taken as the global minimizer.

\section{Numerical results}

The optimal productivity of 1,3-PD and the corresponding optimal control in Problem $\widetilde{\text { NOCP }}$ are obtained by Algorithm 1 on Lenovo DeepComp 1800 PC-cluster Server composed of 16 nodes. Each node is equipped with two Intel $5420 \mathrm{CPU}$ (4 Core, 64-bit, clocked at $2.5 \mathrm{GHz}$ ) and 8 GB memory. The parameters chosen in Algorithm 1, derived empirically after numerous experiments, are listed in Table 2 . The initial state is $x_{0}=(0.102,418.26087,0,0,0)$. The initial terminal time is $T=4$. The initial function $\phi(t)$ is obtained based on [24]. System (23) is solved by using Euler method with a step size of $1 / 3600$.

In Fig. 1, three axes respectively represent the initial concentration of extracellular biomass $x_{01}$, glycerol $x_{02}$, and the value of the cost function with various initial state $x_{01}$ and $x_{02}$. Following the observation of Fig. 1, all the peaks are contained in a polyhedron and the cost function $\widetilde{J}\left(x_{01}, x_{02}, T \mid \sigma\right)$ can reach the maximum value when $\left(x_{01}, x_{02}, T\right) \in$ $\left[a_{1}, b_{1}\right] \times\left[a_{2}, b_{2}\right] \times\left[a_{3}, b_{3}\right]$. Thus, it is confident to draw the conclusion that the cost function $\widetilde{J}\left(x_{01}, x_{02}, T \mid \sigma\right)$ is optimal when $x_{01}$ is $0.9985, x_{02}$ is 404.034 and the optimal terminal time is 4.82 . More accurately, the optimal control and the corresponding cost function for Problem $\widehat{\text { NOCP }}$ are, respectively, $u^{*}=(0.9985,404.034,4.82)$ and $\tilde{J}\left(u^{*} \mid \sigma\right)=81.10$.

The ranges of uncertain system parameter vector perturbations applied to get the results in Figs. 2 and 3 belong to $\left[0.5 \sigma_{k}, 1.5 \sigma_{k}\right], k=1,2, \ldots, 10$. To investigate the cost sensitivity of individual uncertain system parameters $\sigma_{k}, k=$ $1,2, \cdots, 10$, for box plots drew in Fig. 2, we randomly generated 200 perturbations of individual uncertain system parameters $\sigma_{k}, k=1,2, \cdots, 10$, with the initial control strategy $u=(0.102,418.26087,4)$. It turns out that the 7 th component of $\sigma$, i.e., $Y_{2}$, is the most critical system parameter in terms of cost sensitivity: $\left(\partial \tilde{J} / \partial \sigma_{7}\right)^{2}=\left(\partial \tilde{J} / \partial Y_{2}\right)^{2}$ is the dominant term in the sensitivity values in Fig. 2. As another demonstration, we generated the perturbed system 
parameter vector as follows: for $k \neq 7$, we perturbed $\sigma_{k}$ by $50 \%$ (in the negative and positive direction); for $k=7$, we perturbed $\sigma_{7}$ from the disturbance intervals

$$
(0,5 \%),(5 \%, 10 \%), \cdots,(45 \%, 50 \%) .
$$

where the upper limit of each interval is referred to as the "disturbance percentage". For each disturbance percentage $(5 \%, 10 \%, \cdots, 45 \%, 50 \%)$, we generated 200 random system parameter vector according to the above procedure and calculated the corresponding value of the cost sensitivity under $\sigma^{0}$ and initial state vector $x_{0}$ and initial terminal time $T$. Our results are shown as box plots in Fig. 3. From Fig. 3, we can see that the 7th component of $\sigma$, i.e., $Y_{2}$ is the sensitive system parameter.

In our simulations, we generated the perturbed system parameter vector as follows: for $k \neq 7$, we perturbed $\sigma_{k}$ by $10 \%$ (in the negative and positive direction); for $k=7$, we perturbed $\sigma_{7}$ from the disturbance intervals

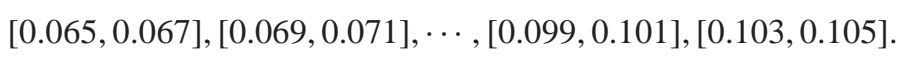

For each disturbance intervals, we generated 1,000 random system parameter vector according to the above procedure and calculated the corresponding value of the cost function under the optimal control strategy $u^{*}$ for including constraint (11) and excluding constraint (11) in Fig. 4. The first thing to note is that the value of the cost function to Problem $\widetilde{\text { NOCP }}$ excluding constraint (11) is very sensitive with respect to changes in $\sigma_{7}$. For example, a change of $2 \times 10^{-3}$ in the value of $\sigma_{7}$ causes the value of the cost function to over $30 \%$. The change in the value of the cost function to Problem $\widetilde{\text { NOCP }}$ including constraint (11), however, is much smaller. The value of the cost function to Problem $\widetilde{\text { NOCP }}$ including constraint (11) under optimal control $u^{*}$ hardly changes when $\sigma_{7}$ is changed.

From Fig. 5, the continuous inequality constraints (12) is satisfied for $t \in[0, T]$.

Under the obtained optimal control strategy, we obtained the optimal yield of 1,3-PD at the optimal terminal time is $81.10 \mathrm{mmol} \mathrm{h}^{-1}$, which is increased by $3.76 \%$ compared to the result $\left(78.16 \mathrm{mmol} \mathrm{h}^{-1}\right)$ in [25]. Compared to the result $\left(41.13 \mathrm{mmol} \mathrm{h}^{-1}\right)$ in [24], our result is increased by $97.2 \%$.

Table 2. The parameters chosen in Algorithm 1.

\begin{tabular}{cccccccccccccc}
\hline$\tau$ & $a_{1}$ & $a_{2}$ & $b_{1}$ & $b_{2}$ & $c_{1}$ & $c_{2}$ & $\epsilon$ & $\tilde{\epsilon}$ & $\tilde{N}$ & $\mathcal{K}$ & $\widetilde{\mathcal{K}}$ & $\varepsilon$ & $\gamma$ \\
\hline 0.26 & 0.01 & 1 & 200 & 1700 & 2 & 10 & 20 & 0.1 & 79 & 600 & 50 & 0.01 & 0.01 \\
\hline
\end{tabular}

\section{Discussions and conclusions}

In this paper, we consider a nonlinear time-delay dynamic system with uncertain system parameters to characterize the process of batch fermentation. To maximize the concentration of 1,3-PD at the terminal time, we introduce an optimal control problem governed by the nonlinear time-delay dynamic system, subject to a cost sensitivity constraint and continuous state inequality constraints. Then, the optimal control problem with varying terminal time is transformed. Using some techniques, we approximate the continuous state inequality constraints by conventional inequality constraints to yield an approximate optimal control problem. A parallel algorithm is proposed to solve the approximate optimal control problem based on the filled function method. Finally, numerical simulation results verified the effectiveness of the numerical solution method.

In fact, optimal control problems involved nonlinear time-delay system appear in various industrial processes. Future studies will generalize the mathematical model to other processes and demonstrate the validity of the numerical solution method. 


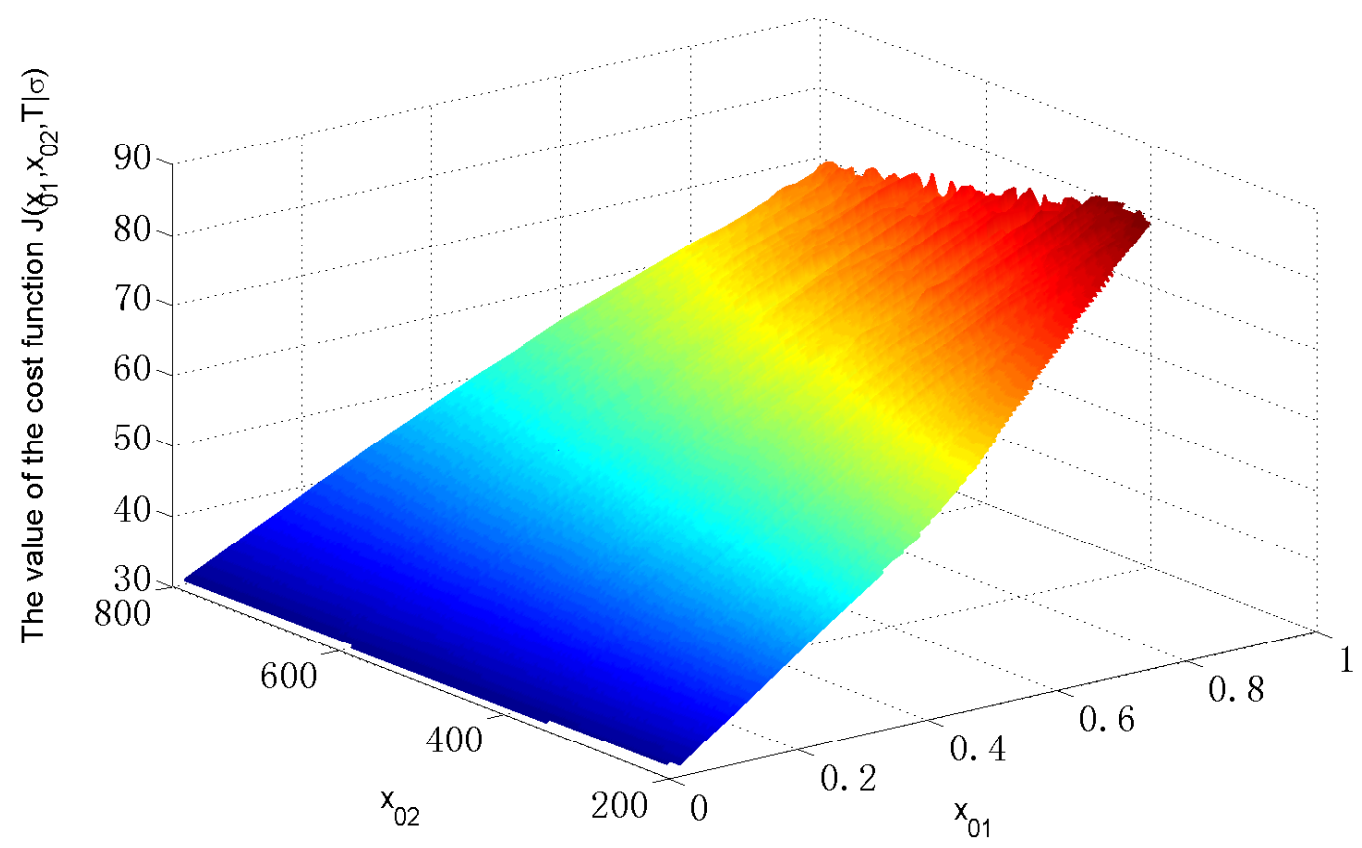

Fig. 1. The value of the cost function $J\left(x_{01}, x_{02}, T \mid \sigma\right)$ for various initial state $x_{01}$ and $x_{02}$. (For interpretation of the references to color in this figure legend, the reader is referred to the web version of this article.)

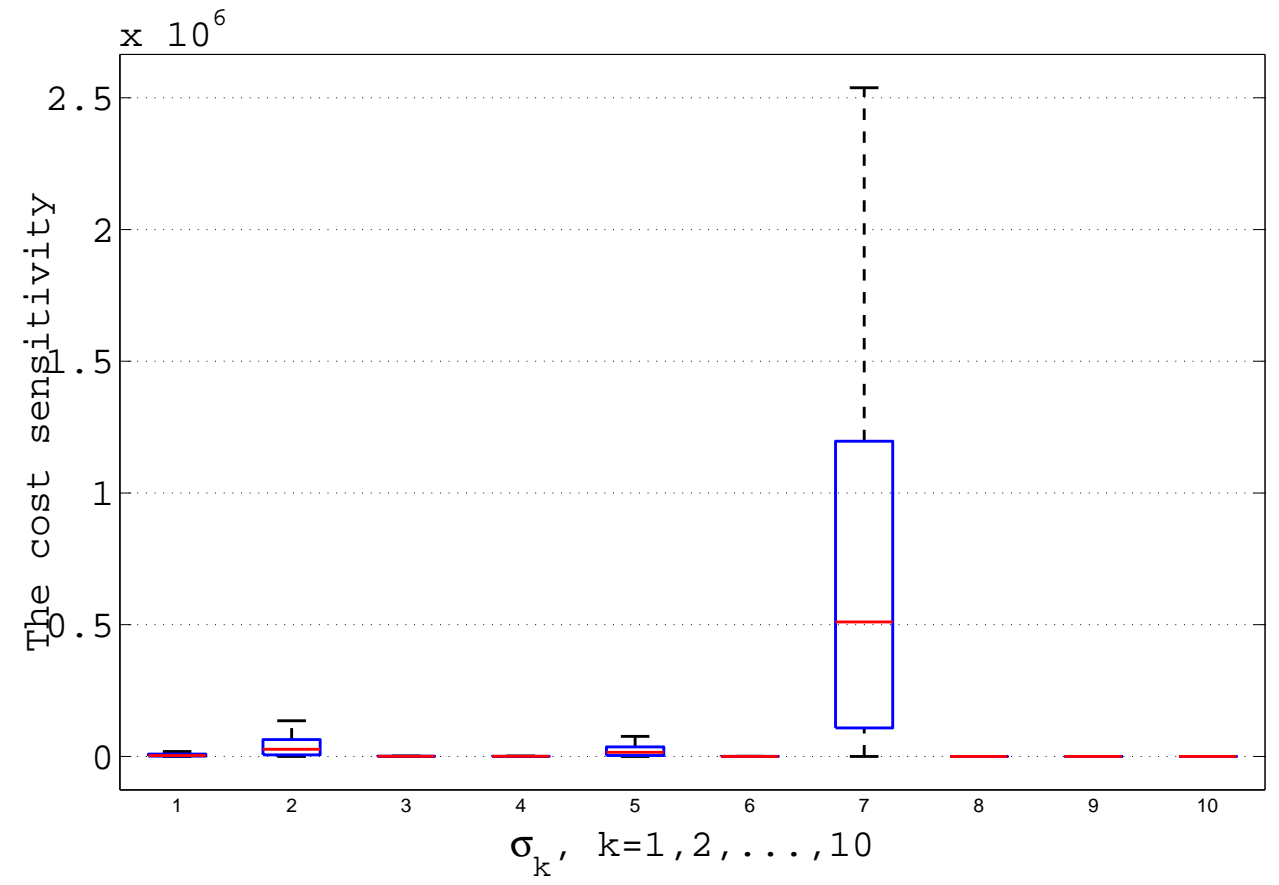

Fig. 2. The cost sensitivity for individual system parameter uncertainty $\sigma_{k}, k=1,2, \cdots, 10$. (For interpretation of the references to color in this figure legend, the reader is referred to the web version of this article.) 


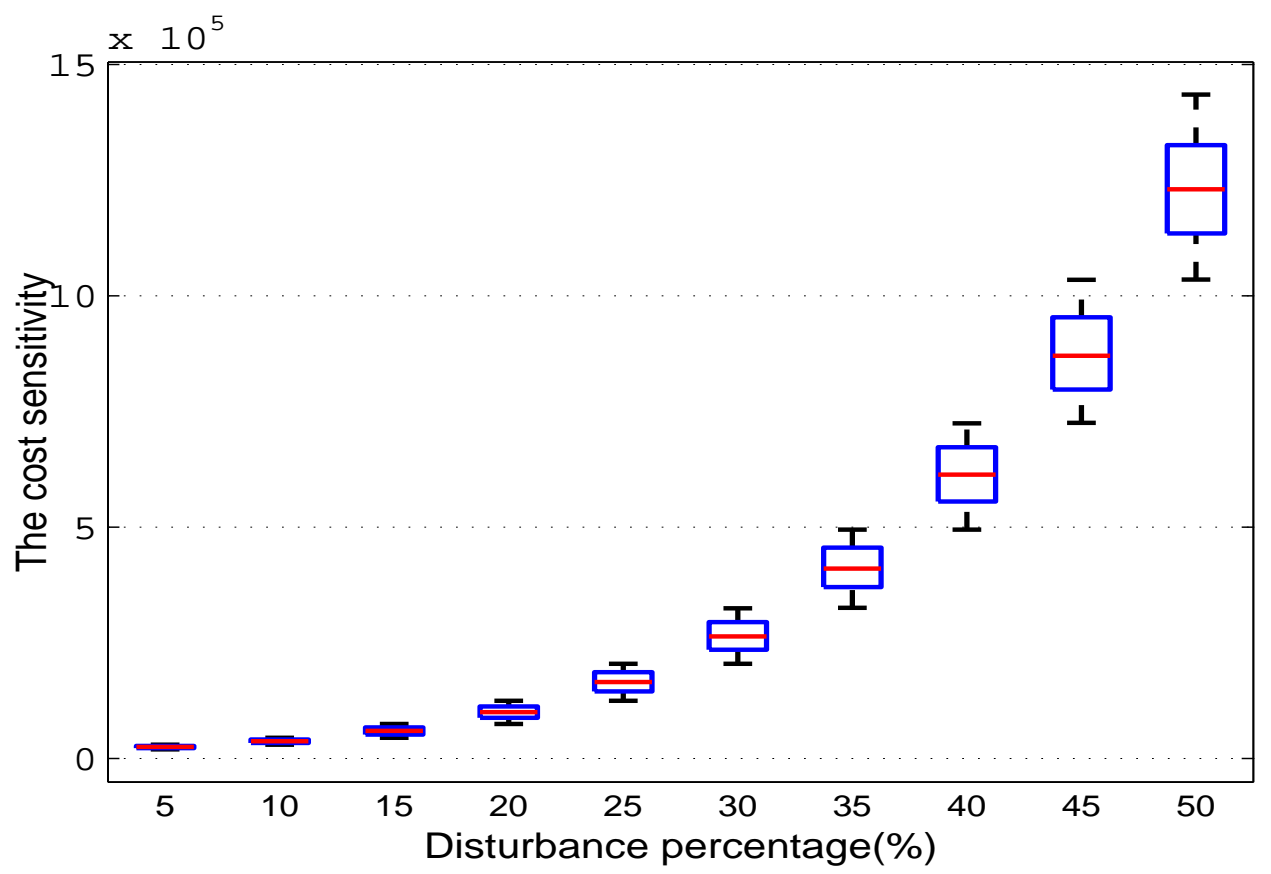

Fig. 3. The cost sensitivity under all uncertain system parameters disturbance. (For interpretation of the references to color in this figure legend, the reader is referred to the web version of this article.)

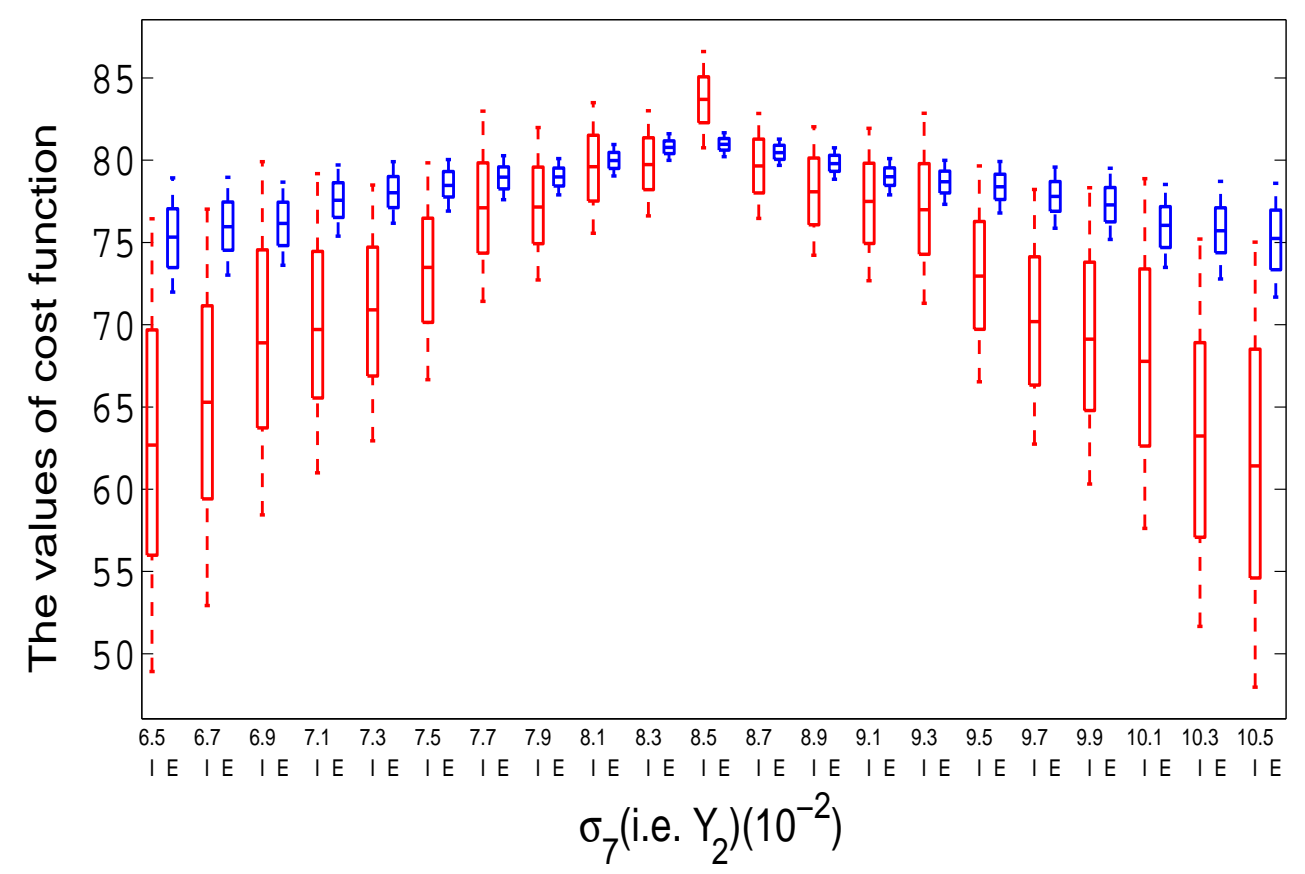

Fig. 4. Variation in the value of the cost function due to the variation of $\sigma_{7}\left(Y_{2}\right) . I:$ (red) Problem OCP including (11), $E$ : (blue) Problem OCP excluding (11). (For interpretation of the references to color in this figure legend, the reader is referred to the web version of this article.) 

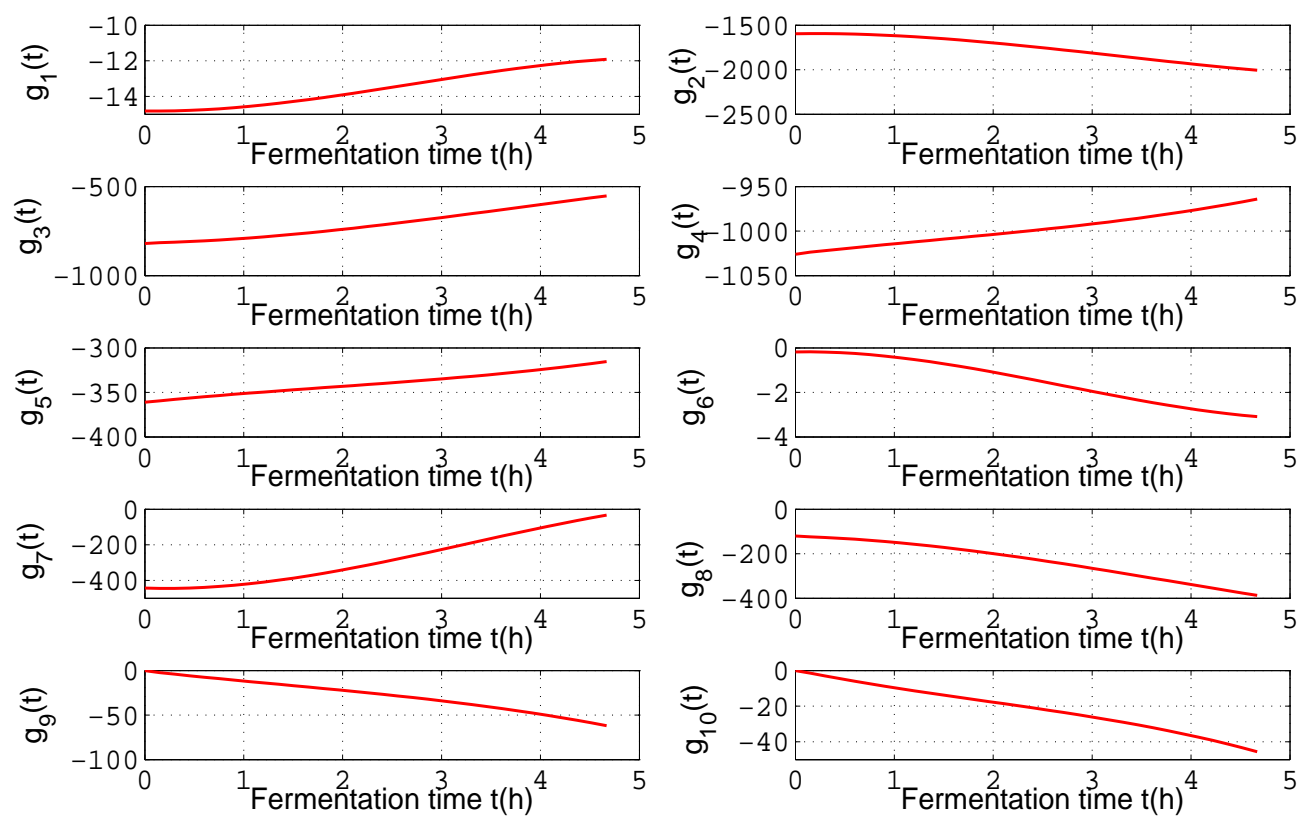

Fig. 5. $g_{j}(t)=x_{j}\left(t \mid u^{*}, \sigma\right)-x_{j}^{*}, g_{j+5}(t)=x_{j *}-x_{j}\left(t \mid u^{*}, \sigma\right), j \in I_{5}$, under $u^{*}=\left[x_{01}^{*}, x_{02}^{*}, T^{*}\right]$ in batch culture process in $t \in\left[0, T^{*}\right]$.

\section{Acknowledgements}

This work was supported by the National Natural Science Foundation of China (Grant Nos. 11171050 and 11371164), the National Science Foundation for the Youth of China (Grant Nos. 11301051, 11301081, 11401073 and 11501574), the Provincial Natural Science Foundation of Fujian (Grant No. 2014J05001), the Fundamental Research Funds for Central Universities in China (Grant No. DUT15LK25), Natural Science Foundation of Shandong Province in China (Grant No. ZR2015AL010) and the China Scholorship Council (CSC, No. 201506060121).

\section{References}

[1] H. Biebl, K. Menzel, A.P. Zeng, W.D. Deckwer, Microbial production of 1, 3-propanediol, Appl. Microbiol. Biot. 52(3)(1999)289-297

[2] H. Biebl, A.P. Zeng, K. Menzel, W.D. Deckwer, Fermentation of glycerol to 1, 3-propanediol and 2, 3-butanediol by Klebsiella pneumoniae, Appl. Microbiol. Biot. 50(1)(1998)24-29

[3] A.P. Zeng, H. Biebl, Bulk chemicals from biotechnology: the case of 1, 3-propanediol production and the new trends. In Tools and Applications of Biochemical Engineering Science, Springer Berlin Heidelberg, (2002)239-259

[4] K. Ahrens, K. Menzel, A.P. Zeng, W.D. Deckwer, Kinetic, dynamic, and pathway studies of glycerol metabolism by Klebsiella pneumoniae in anaerobic continuous culture: III. Enzymes and fluxes of glycerol dissimilation and 1,3-propanediol formation, Biotechnol. Bioeng. $59(5)(1998) 544-552$

[5] C.E. Nakamura, G.M. Whited, Metabolic engineering for the microbial production of 1,3-propanediol, Curr. Opin. Biotechnol. 14(15)(2003)454-459

[6] A.P. Zeng, H. Biebl, Bulk chemicals from biotechnology: the case of 1,3-propanediol production and the new trends, Adv. Biochem. Eng. Biotechnol. 74(2002)239-259

[7] K. Menzel, A.P. Zeng, W.D. Deckwer, High concentration and productivity of 1, 3-propanediol from continuous fermentation of glycerol by Klebsiella pneumoniae, Enzyme Microb. Tech. 20(2)(1997)82-86.

[8] L. Wang, Determining the transport mechanism of an enzyme-catalytic complex metabolic network based on biological robustness, Bioproc. Biosyst. Eng. 36(4)(2013)433-441.

[9] A. Ashoori, B. Moshiri, A. Khaki-Sedigh, M. R. Bakhtiari, Optimal control of a nonlinear fed-batch fermentation process using model predictive approach, J. Process Contr. 19(7)(2009)1162-1173

[10] L. Wang, Q. Lin, R. Loxton, K.L. Teo, G. Cheng, Optimal 1, 3-propanediol production: Exploring the trade-off between process yield and feeding rate variation, J. Process Contr. 32(2015)1-9. 
[11] R.K. Saxena, P. Anand, S. Saran, J. Isar, Microbial production of 1,3-propanediol: Recent developments and emerging opportunities, Biotechnol. Adv. 27 (2009) 895-913

[12] A.P. Zeng, A kinetic model for product formation of microbial and mammalian cells, Biotechnol. Bioeng. 46(4)(1995)314-324

[13] J.L. Yuan, X. Zhu, X. Zhang, H.C. Yin, E.M. Feng, Z.L. Xiu, Robust identification of enzymatic nonlinear dynamical systems for 1,3propanediol transport mechanisms in microbial batch culture, Appl. Math. Comput. 232(2014)150-163

[14] J.L. Yuan, X. Zhang, X. Zhu, E.M. Feng, H.C. Yin, Z.L. Xiu, Modelling and pathway identification involving the transport mechanism of a complex metabolic system in batch culture, Commun. Nonlinear Sci. Numer. Simulat. 19(16)(2014)2088-2103

[15] Z.G. Jiang, J.L. Yuan, E.M. Feng, Robust identification and its properties of nonlinear bilevel multi-stage dynamic system, Appl. Math. Comput. 219(12)(2013)6979-6985

[16] H. Yin, J.L. Yuan, X. Zhang, J. Xie, E. Feng, Z. Xiu, Modeling and parameter identification for a nonlinear multi-stage system for dha regulon in batch culture, Appl. Math. Modell. 40(2016)468-484

[17] J.L. Yuan, X. Zhang, X. Zhu, H.C. Yin, E.M. Feng, Z.L. Xiu, B. Tan, Identification and robustness analysis of nonlinear multi-stage enzymecatalytic dynamical system in batch culture, Comp. Appl. Math. 34(2015)957-978

[18] J.L. Yuan, L. Wang, X. Zhang, E. Feng, H. Yin, Z. Xiu, Parameter identification for a nonlinear enzyme-catalytic dynamic system with time-delays, J. Glob. Optim. 62(2015)791-810

[19] J.L. Yuan, X. Zhang, X. Zhu, E. Feng, H. Yin, Z. Xiu, Pathway identification using parallel optimization for a nonlinear hybrid system in batch culture, Nonlinear Analysis: Hybrid Sys. 15 (2015) 112-131

[20] J. Wang, J. Ye, E.M. Feng, H. Yin, Z. Xiu, Modeling and identification of a nonlinear hybrid dynamical system in batch fermentation of glycerol, Math. Comput. Model. 54(2011)618-624

[21] Y. Wang, L. Wang, Y. Zhao, A. Song, Y. Ma, A stochastic model for microbial fermentation process under Gaussian white noise environment, Numer. Algebra Control Optim. 5(4)(2015)381-392.

[22] X. Zhu, E.M. Feng, Joint estimation in batch culture by using unscented kalman filter, Biotechnol. Bioproc. Engineering. 17(6)(2012)12381243.

[23] G.M. Cheng, L. Wang, R. Loxton, Q. Lin, Robust optimal control of a micro-bial batch culture process, J. Optim. Theory. Appl. 161(1)(2014)342-362

[24] C.Y. Liu, Modelling and parameter identification for a nonlinear time-delay system in microbial batch fermentation, Appl. Math. Model 37 (2013) 6899-6908

[25] Y.S. Yu, Optimal control of a nonlinear time-delay system in batch fermentation process, Math. Probl. Eng. (2014), [Article ID 478081, 7 pages], http://dx.doi.org/10.1155/2014/478081

[26] V. Rehbock, K.L. Teo, L.S. Jennings, A computational procedure for suboptimal robust controls, Dynam. Control 2(1992)331-348

[27] W. Wei, K.L. Teo, Z. Zhan, A numerical method for an optimal control problem with minimum sensitivity on coefficient variation, Appl. Math. Comput. 218(2011)1180-1190

[28] R. Loxton, K.L. Teo, V. Rehbock, Robust suboptimal control of nonlinear systems, Appl. Math. Comput. 217(2011)6566-6576

[29] R. Loxton, Q. Lin, K.L. Teo, Switching time optimization for nonlinear switched systems: Direct optimization and the time-scaling transformation, Pac. J. Optim. 10(3)(2014)537-560

[30] Q. Lin, R. Loxton, K.L. Teo, The control parameterization method for nonlinear optimal control: a survey, J. Ind. Manag. Optimi. 10(1)(2014)275-309.

[31] C. Liu, R. Loxton, K.L. Teo, A computational method for solving time-delay optimal control problems with free terminal time, Syst. Control Let. 72 (2014)53-60.

[32] E. Polak, Optimization algorithms and consistent approximations. Springer-Verlag, New York 1997

[33] R.P. Ge, Y.F. Qin, A class of filled functions for finding global minimizers of a function of several variables, J. Optim. Theor. Appl. 54(2)(1987)241-252

[34] Y. Gao, Y. Yang, M. You, A new filled function method for global optimization, Appl. Math. Comput. 268(2015)685-695

[35] H. Lin, Y. Gao, Y. Wang, A continuously differentiable filled function method for global optimization, Numer. Algor. 66(2014)511-523

[36] Q.Q. Chai, R. Loxton, K.L. Teo, C.H. Yang, A unified parameter identification method for nonlinear time-delay systems, J. Ind. Manag. Optim. 9(2)(2013)471-486

[37] C. Yu, Q. Lin, R. Loxton, K.L. Teo, G. Wang, A hybrid time-scaling transformation for time-delay optimal control problems, J. Optimiz. Theory App. Doi 10.1007/s10957-015-0783-z (2015) 